\title{
A Wolf-Rayet-like progenitor of SN 2013cu from spectral observations of a stellar wind
}


A. Horesh $^{4}$, A. De Cia ${ }^{1}$, F. Taddia ${ }^{6}$, J. Sollerman ${ }^{6}$, D. Perley ${ }^{4}$, P. M. Vreeswijk ${ }^{1}$, S. R. Kulkarni ${ }^{4}$, P. E. Nugent ${ }^{7}$, A. V. Filippenko $^{8}$ \& J. C. Wheeler ${ }^{5}$

The explosive fate of massive Wolf-Rayet $\operatorname{stars}^{1}$ (WRSs) is a key open question in stellar physics. An appealing option is that hydrogen-deficient WRSs are the progenitors of some hydrogen-poor supernova explosions of types IIb, Ib and Ic (ref. 2). A blue object, having luminosity and colours consistent with those of some WRSs, has recently been identified in pre-explosion images at the location of a supernova of type Ib (ref. 3), but has not yet been conclusively determined to have been the progenitor. Similar work has so far only resulted in non-detections ${ }^{4}$. Comparison of early photometric observations of type Ic supernovae with theoretical models suggests that the progenitor stars had radii of less than $10^{12}$ centimetres, as expected for some WRSs ${ }^{5}$. The signature of WRSs, their emission line spectra, cannot be probed by such studies. Here we report the detection of strong emission lines in a spectrum of type IIb supernova 2013cu (iPTF13ast) obtained approximately 15.5 hours after explosion (by 'flash spectroscopy', which captures the effects of the supernova explosion shock breakout flash on material surrounding the progenitor star). We identify Wolf-Rayet-like wind signatures, suggesting a progenitor of the $\mathrm{WN}(\mathrm{h})$ subclass (those WRSs with winds dominated by helium and nitrogen, with traces of hydrogen). The extent of this dense wind may indicate increased mass loss from the progenitor shortly before its explosion, consistent with recent theoretical predictions ${ }^{6}$.

Wolf-Rayet stars are massive stars stripped of their outer, hydrogenrich envelope. These stars blow strong, hydrogen-poor winds. The inner part of the wind engulfing the star is dense and optically thick, and efficiently absorbs the ionizing continuum from the hot stellar surface. Farther from the star, the density drops and the wind becomes optically thin in the continuum, leading to a rich emission spectrum of recombination lines. Detailed models of such spectra can be constructed ${ }^{1,7}$ and depend essentially on only three parameters: the effective temperature, $T_{\text {eff }}$, in the line-forming region, a normalized radius, $R_{\mathrm{t}}$ (a combination of the stellar radius, luminosity, mass loss rate and wind terminal velocity ${ }^{7}$ ), and the chemical composition, $Z$, of the wind (assumed to be uniform, spherical and of a constant mass loss rate). The composition of the wind determines Wolf-Rayet spectral classes: stars with dominant $\mathrm{He}$ and $\mathrm{N}$ lines belong to the WN class (with those also showing traces of $\mathrm{H}$ usually denoted as $\mathrm{WN}(\mathrm{h})$ ), stars with strong carbon lines belong to the WC class and rare (and possibly hotter) stars with oxygen-rich spectra belong to the WO class ${ }^{1}$.

Shortly after a WRS explodes as a supernova, the outer parts of the wind (which in some cases ${ }^{1}$ extend to radii of $>10^{13} \mathrm{~cm}$ ) that have not yet been swept up by the expanding supernova ejecta will emit strong recombination lines in response to ionizing flux released by the explosion shock breakout from the stellar surface. We estimate an increase in ionizing luminosity by a factor of order $10^{2}-10^{4}$, assuming an initial absolute magnitude range of $-2.5 \mathrm{mag}<M<-10 \mathrm{mag}$ for the exploding $\mathrm{WRS}^{1}$ and a typical early-time luminosity of $M=-12.5 \mathrm{mag}$ for the resulting supernova $^{3,5,8}$. The effective temperature, $T_{\text {eff, will also change, being }}$ very high $\left(>10^{5} \mathrm{~K}\right)$ shortly after explosion ${ }^{9}$ and decreasing as the shocked supernova ejecta cool. The radiation illuminating the surviving Wolf-Rayet wind will thus effectively vary monotonically through the range of temperatures in WRS line-forming regions. Because the wind parameters (composition, mass loss rate and terminal velocity) do not change, the measured line spectrum observed shortly after explosion should be similar to that of a WRS with the spectral class of the exploding progenitor (because the spectral classes mainly reflect the wind composition). The high wind densities around WRSs (with electron densities of $n_{\mathrm{e}}=10^{11}-10^{12} \mathrm{~cm}^{-3}$ ) imply short recombination times ${ }^{10}$, $t_{\text {rec }} \approx 3.9 \times 10^{12}\left(n_{\mathrm{e}} / \mathrm{cm}^{-3}\right)^{-1}\left(T / 10^{4} \mathrm{~K}\right)^{0.85} \mathrm{~s}$, typically of a few minutes for Wolf-Rayet densities and temperatures, and so the emitted spectrum will promptly react to the rapidly evolving supernova radiation field.

We obtained rapid spectroscopic observations of the recent type IIb supernova SN 2013cu (iPTF13ast) shortly after shock breakout (by flash spectroscopy; see Methods). This event was first detected by the Intermediate Palomar Transient Factory (iPTF) survey ${ }^{11}$ on 2013 May 3.18 (we express dates in coordinated universal time (UTC)), photometrically confirmed $5.8 \mathrm{~h}$ later and promptly identified by an on-duty astronomer who triggered rapid follow-up observations ${ }^{12}$, including an optical spectrum obtained just $4 \mathrm{~h}$ later. Analysis of the early-time light curve of this supernova (Extended Data Fig. 1) suggests that it exploded on May 2.93, implying that the first iPTF detection and the first spectrum correspond to only $5.7 \mathrm{~h}$ and $15.5 \mathrm{~h}$ after the explosion, respectively. A full description of the supernova and its evolution will be reported in a forthcoming publication (A.G.-Y. et al., manuscript in preparation). We note that this event was independently observed by the MASTER survey on May 5.3 ( $\sim 2.3 \mathrm{~d}$ after explosion), and it was assigned the name SN 2013cu following spectroscopic confirmation ${ }^{13}$.

Our first spectrum of SN 2013cu reveals a continuum and emission lines that bear a striking resemblance to spectra of WRSs (Fig. 1a). According to accepted Wolf-Rayet terminology ${ }^{1}$, the spectrum is classified as subclass WN6(h) (Fig. 1a, blue trace); the relative strength of nitrogen to carbon lines precludes a WC classification, and the absence of any high-excitation oxygen lines is inconsistent with a WO star. The stronger lines ( $\mathrm{H} \alpha, \mathrm{H} \beta, \mathrm{N}$ IV $\lambda 7,115$ (7,115 $\AA$ wavelength) and He II $\lambda 5,411$ ) exhibit a complex profile (Fig. 2) consisting of a relatively broad base $\left(\sim 2,500 \mathrm{~km} \mathrm{~s}^{-1}\right.$ full width at zero intensity (FWZI)) on which prominent narrow, unresolved lines $(F W Z I \approx 3 \AA$; velocity dispersion, $<150 \mathrm{~km} \mathrm{~s}^{-1}$ ) are superimposed. This is consistent with predictions for Wolf-Rayet pre-supernova wind velocities ${ }^{14,15}$, although we cannot exclude the possibility that at least some of the observed line broadening is produced by electron scattering rather than genuine velocity dispersion. To the best of our knowledge, no similar spectra of a stripped (H-poor) supernova have been acquired previously. Wolf-Rayet-like 



spectroscopic features have been observed in spectra of some H-rich (non-stripped) supernovae obtained at substantially later epochs, and their typically much broader lines were interpreted as emerging from interaction with circumstellar material ${ }^{16}(\mathrm{CSM})$. We further discuss these previous observations in Extended Data Fig. 3.

We analyse our very early spectrum using the PoWR grid of WolfRayet spectral models ${ }^{7}$ (http://www.astro.physik.uni-potsdam.de/ wrh/ PoWR/powrgrid1.html). We find an excellent fit with $\mathrm{WN}(\mathrm{h})$ models calculated assuming an $\mathrm{H}$ fraction of $20 \%$ (by mass) and a temperature of
Figure 1 | Spectroscopy of the type IIb supernova SN 2013cu reveals transient Wolf-Rayet-like features. a, The early spectrum of SN 2013cu (black; specific flux, $F_{\lambda}$, versus wavelength) is compared with models of WNL-class WRSs ${ }^{7}$ (red and grey), showing remarkable similarity in continuum shape and in line features (strong He, N and Balmer lines indicate a WN6(h) classification; compare with HD 192163 (blue)). b. Emission line evolution during the first week. The first spectrum (red) is compared with later spectra. By day 3 (blue and cyan), the initially strong Wolf-Rayet features disappear, but the $\mathrm{H} \alpha$ line remains constant at $3.4 \times 10^{-15} \mathrm{erg} \mathrm{s}^{-1} \mathrm{~cm}^{-2}$. The spectrum on day 6 (magenta) is almost featureless, except for weak $\mathrm{H} \alpha$ emission (inset) with an intensity less than a tenth that of day 3 , probably because the line-forming region has been cleared by the expanding ejecta. c, SN 2013cu is of type IIb. A spectrum of SN 2013cu 69 days after explosion (black) is compared with the prototypical type IIb supernovae SN 1993J (60 days; red) and SN 2011dh (43 days; green), and with the typical non-stripped type II-P supernova SN 2004et (45 days; blue). SN 2013cu, SN 2011dh and SN 1993J exhibit strong blue-shifted He I absorption at 5,876, 6,678, 7,065 and 7,281 $\AA$ (marked with black vertical lines), which are not detected in type II-P supernovae. See Methods for more details.

around $50,000 \mathrm{~K}$. This temperature is consistent with the lower limit obtained from early ultraviolet photometry from NASA's Swift spacecraft (Extended Data Fig. 1). The essentially perfect match of the observed and modelled continuum shapes indicates that dust reddening must be negligible; any pre-existing dust must have been destroyed by the supernova explosion flash (Methods). We note that among the large catalogue of Galactic WN stars specifically modelled in this manner, no stars drive winds that require more than $56 \% \mathrm{H}$ by mass. Presumably, custom spectral fits ${ }^{7}$ could be calculated and used to determine more accurately the physical parameters of the detected Wolf-Rayet wind. Assuming that the spectrum was obtained $15.5 \mathrm{~h}$ after explosion and that a standard ejecta velocity of $10^{4} \mathrm{~km} \mathrm{~s}^{-1}$ is applicable, the narrowline-emitting material must be located at radii greater than $\sim 5 \times 10^{13} \mathrm{~cm}$ for it not to have been swept up and accelerated by the expanding ejecta. This lower limit is consistent with the extent of some Wolf-Rayet winds, where the line-formation region extends out to several (five to ten) times ${ }^{17}$ the hydrostatic radius. Recent pre-supernova Wolf-Rayet models ${ }^{14}$ suggest hydrostatic radii of $10-20$ solar radii for WN supernova progenitors, consistent with line-formation regions extending to several hundred solar radii $^{1}$, or $>10^{13} \mathrm{~cm}$.

We further constrain the physical location of the wind using the following calculation. We measure the $\mathrm{H} \alpha$ line flux from our spectrum (calibrated to our host-subtracted photometry) and obtain $F_{\mathrm{H} \alpha}=3.4 \times$ $10^{-15} \mathrm{erg} \mathrm{s}^{-1} \mathrm{~cm}^{-2}$. We translate this to line luminosity using a luminosity distance to the host galaxy, UGC 9379, of $d=108 \mathrm{Mpc}$ (calculated for a flat $\Lambda$ cold dark matter cosmology with Hubble constant $H_{0}=$ $73 \mathrm{~km} \mathrm{~s}^{-1} \mathrm{Mpc}^{-1}$, a matter density of $\Omega_{\mathrm{m}}=0.27$ and a redshift $z=$ 0.025734, obtained from the NASA Extragalactic Database (NED), as well as negligible extinction), obtaining $L_{\mathrm{H} \alpha}=4.8 \times 10^{39} \mathrm{erg} \mathrm{s}^{-1}$. We can then estimate ${ }^{18}$ the pre-explosion $\mathrm{H}$ mass loss rate

$$
\begin{aligned}
\dot{M}= & 0.01\left(L_{\mathrm{H} \alpha} /\left(2 \times 10^{39} \mathrm{erg} \mathrm{s}^{-1}\right)\right)^{1 / 2} \\
& \times\left(v_{\mathrm{w}} /\left(500 \mathrm{~km} \mathrm{~s}^{-1}\right)\right)\left(r /\left(10^{15} \mathrm{~cm}\right)\right)^{1 / 2} M_{\odot} \mathrm{yr}^{-1}
\end{aligned}
$$

assuming that the lines are formed at a radius $r$ by recombination, that the width of the emitting shell is similar to its radius, that the explosion is spherical symmetric and that the density of the wind decreases as $r^{-2}$. We assume a wind velocity of $v_{\mathrm{w}}=2,500 \mathrm{~km} \mathrm{~s}^{-1}$, consistent with our spectra and as expected for WRSs, but our results are not sensitive to this value and remain essentially unchanged for velocities in the range $100<v_{\mathrm{w}}<2,500 \mathrm{~km} \mathrm{~s}^{-1}$.

We check for self-consistency by calculating the electron density and, hence, the Thomson optical depth

$$
\begin{aligned}
\tau= & 0.3\left(\dot{M} /\left(0.01 M_{\odot} \mathrm{yr}^{-1}\right)\right) \\
& \times\left(v_{\mathrm{w}} /\left(500 \mathrm{~km} \mathrm{~s}^{-1}\right)\right)^{-1}\left(r /\left(10^{15} \mathrm{~cm}\right)\right)^{-1}
\end{aligned}
$$




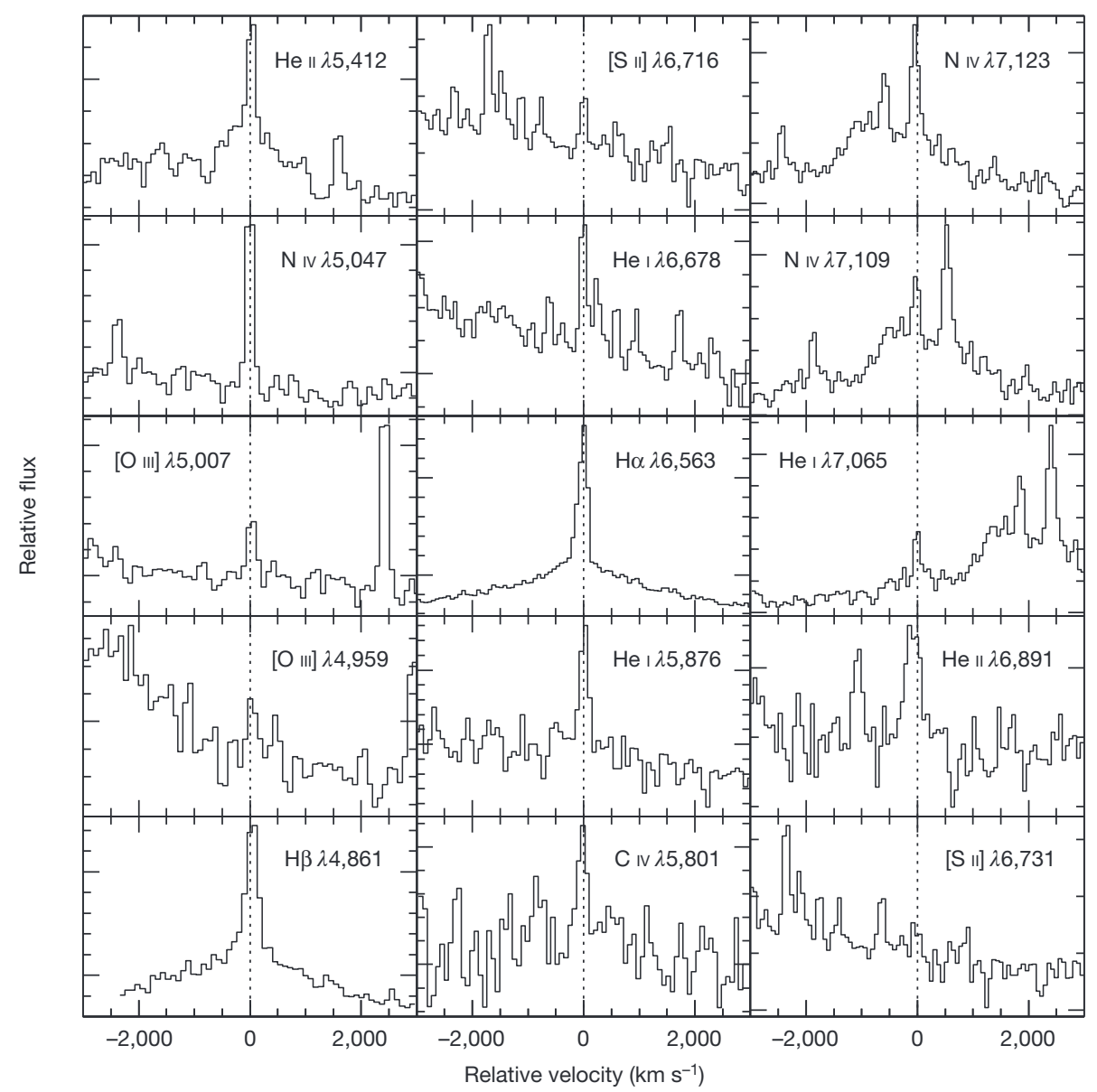

Figure $2 \mid$ Emission line velocity structure at $\mathbf{1 5 . 5} \mathbf{h}$. The strongest lines $(\mathrm{H} \alpha, \mathrm{H} \beta, \mathrm{He}$ II $\lambda 5,411$ and the N IV $\lambda 7,115$ complex) show broad wings

expected at this radius, and require it to be lower than $\tau=1$ for the line emission to escape. We find that this self-consistency requirement places a lower limit of $r=2 \times 10^{14} \mathrm{~cm}$ on the radius of the line-formation region, and implies substantial mass loss rates, $\dot{M} \mathrm{w} 0.03 M_{\odot} \mathrm{yr}^{-1}$. If we interpret the disappearance of essentially all emission lines from our day- 6 spectrum (Fig. 1b) as evidence that the wind was swept up by the expanding ejecta (moving at $10^{4} \mathrm{~km} \mathrm{~s}^{-1}$ ), the radius of the line-emitting region must be $r<5.2 \times 10^{14} \mathrm{~cm}$, which is fully consistent with our estimates.

We can then calculate the total $\mathrm{H}$ mass by integrating over $r$ :

$$
\begin{aligned}
M_{\mathrm{tot}}= & 0.006\left(\dot{M} /\left(0.01 M_{\odot} \mathrm{yr}^{-1}\right)\right) \\
& \times\left(v_{\mathrm{w}} /\left(500 \mathrm{~km} \mathrm{~s}^{-1}\right)\right)^{-1}\left(r /\left(10^{15} \mathrm{~cm}\right)\right)^{-1} M_{\odot}
\end{aligned}
$$

This indicates a range of $0.008 M_{\odot} \mathrm{V} M_{\mathrm{tot}} \mathrm{V} \quad 0.0035 M_{\odot}$ for the range of permitted $\mathrm{H}$ masses. Assuming that the typical $\mathrm{H}$ abundance for $\mathrm{WN}(\mathrm{h})$ stars ( 20\%) applies, the total wind mass (dominated by $\mathrm{He}$ ) can be estimated to be several times larger than these values. Detailed simulations ${ }^{19}$ show that as little as $0.1 M_{\odot}$ of He-dominated CSM would result in strong spectroscopic interaction signatures (that we do not observe), consistent with our derived total masses.

We conclude that we have directly detected a Wolf-Rayet-like wind from the supernova progenitor with a $\mathrm{WN}(\mathrm{h})$ spectral class, indicating a low $\mathrm{H}$ mass fraction. Assuming that the wind composition we measure represents the surface composition of the progenitor star, our observations indicate that some members of the spectroscopic WN(h) WolfRayet class explode after having lost most of their hydrogen envelope, exposing the CNO-processed, N-rich He layer below. Analysis of photometric and spectroscopic follow-up observations (A.G.-Y. et al., manuscript in preparation) confirms that the explosion was indeed a extending out to $\sim 2,500 \mathrm{~km} \mathrm{~s}^{-1}$. Other weaker lines are narrow and unresolved.

supernova of type IIb (Fig. 1c), as expected if the progenitor was a massive star that lost all but $\sim 0.1 M_{\odot}$ of its $\mathrm{H}$ envelope ${ }^{20}$.

Our observations have interesting implications. First, we note that the derived values of the mass loss rate and emission-line-region size are quite extreme compared with known Wolf-Rayet observations and radiatively driven models ${ }^{21}$, including models with clumpy, inflated atmospheres ${ }^{22}$. This suggests that the mass loss rate from the progenitor star may have increased shortly (of order $1 \mathrm{yr}$ for the assumed velocities) before its explosion. Interestingly, such pre-supernova activity may be explained by recent wave-driven models ${ }^{6}$, or a more extreme envelope inflation ${ }^{22}$ may be indicated. These data can thus provide a key diagnostic of the final stages of nuclear core burning in massive stars, which are currently poorly understood, with possible implications for the explosion mechanism itself. In any case, the star probably exploded inside a thick wind, and the explosion shock may have broken out from the opaque inner wind rather than from the hydrostatic surface of the star?.

Our finding is in general accord with some previous work on type IIb supernova progenitors. Direct imaging of the progenitor of SN 2008ax (ref. 23) is consistent with a WN(h) progenitor. Furthermore, increased mass loss during the final year before explosion may inflate the apparent photospheric radius of the pre-supernova star, making stars with compact cores appear to have extended (low-mass) envelopes ${ }^{22}$ and possibly reconciling the conflicting findings about the progenitor of the type IIb supernova SN 2011dh (refs 24-27). Regardless of the exact mechanism, our observations suggest that substantial Wolf-Rayet-like winds pre-date at least some type IIb supernovae. A strong metallicity dependence of this process may explain the trend in the type IIb/type Ib supernova number ratio with host-galaxy metallicity ${ }^{28}$. Future studies of numerous additional supernova progenitors via their spectroscopic 
wind signatures (Methods) would provide powerful constraints on the final stages of massive-star evolution.

\section{METHODS SUMMARY}

Photometry. The iPTF survey telescope was used to obtain $r$-band observations. Photometry was measured using our custom pipeline performing point spread function (PSF) photometry on iPTF images after removing a reference image constructed from pre-explosion data using image subtraction. Swift ultraviolet absolute AB magnitudes (Extended Data Fig. 1) were measured using standard pipeline reduction and were corrected for host-galaxy contamination using late-time Swift images. Spectroscopy. Our earliest $(15.5 \mathrm{~h})$ and latest $(69 \mathrm{~d})$ spectra were obtained using the DEIMOS spectrograph mounted on the Keck II 10-m telescope using the grating with 600 lines per millimetre and an exposure time of $600 \mathrm{~s}$. Additional spectra were obtained using ALFOSC mounted on the 2.56- $\mathrm{m}$ NOT telescope, LRS mounted on the 10.4-m HET telescope (day3), and LRIS mounted on the Keck I 10-m telescope (day 6). All spectra were reduced using standard pipelines and are digitally available on WISeREP ${ }^{29}$. The method of flash spectroscopy is described in detail in Methods.

Online Content Any additional Methods, Extended Data display items and Source Data are available in the online version of the paper; references unique to these sections appear only in the online paper.

\section{Received 23 October 2013; accepted 25 March 2014.}

1. Crowther,P. A. Physical properties of Wolf-Rayet stars. Annu. Rev. Astron. Astrophys. 45, 177-219 (2007)

2. Filippenko, A. V. Optical spectra of supernovae. Annu. Rev. Astron. Astrophys. 35, 309-355 (1997)

3. Cao, Y. et al. Discovery, rise and progenitor of a stripped envelope supernova iPTF13bvn. Astrophys. J. 775, L7 (2013).

4. Eldridge, J. J., Fraser, M., Smartt, S. J., Maund, J. R. \& Crockett, R. M. The death of massive stars - II. Observational constraints on the progenitors of type lbc supernovae. Mon. Not. R. Astron. Soc. 436, 774-795 (2013)

5. Corsi, A. et al. Evidence for a compact Wolf-Rayet progenitor for the type lc supernova PTF 10vgv. Astrophys. J. 747, L5 (2012).

6. Shiode, J. H. \& Quataert, E. Setting the stage for circumstellar interaction in core-collapse supernovae Il: wave-driven mass loss in supernova progenitors. Astrophys. J. 780, 96 (2014).

7. Hamann, W.-R., Gräfener, G. \& Liermann, A. The Galactic WN stars. Spectral analyses with line-blanketed model atmospheres versus stellar evolution models with and without rotation. Astron. Astrophys. 457, 1015-1031 (2006).

8. Dessart, L. et al. Core-collapse explosions of Wolf-Rayet stars and the connection to type Ilb/lb/lc supernovae. Mon. Not. R. Astron. Soc. 414, 2985-3005 (2011).

9. Ofek, E. O. et al. Supernova PTF 09UJ: a possible shock breakout from a dense circumstellar wind. Astrophys. J. 724, 1396-1401 (2010)

10. Osterbrock, D. E. \& Ferland, G. J. Astrophysics of Gaseous Nebulae and Active Galactic Nuclei 47 (University Science Books, 2006).

11. Law, N. M. et al. The Palomar Transient Factory: system overview, performance, and first results. Publ. Astron. Soc. Pacif. 121, 1395-1408 (2009).

12. Gal-Yam, A. et al. Real-time detection and rapid multiwavelength follow-up observations of a highly subluminous type II-P supernova from the Palomar Transient Factory survey. Astrophys. J. 736, 159 (2011).

13. Denisenko, D. et al. Supernova 2013cu in UGC $9379=$ PSN J14335897+4014207. Central Bur. Electr. Telegr. abstr. 3540 (2013).

14. Groh, J. H., Meynet, G., Georgy, C. \& Ekstrom, S. Fundamental properties of core-collapse supernova and GRB progenitors: predicting the look of massive stars before death. Astron. Astrophys. 558, A131 (2013)

15. Niedzielski, A. \& Skorzynski, W. Kinematical structure of Wolf-Rayet winds. 1. Terminal wind velocity. Acta Astron. 52, 81-104 (2002)

16. Leonard, D. C., Filippenko, A. V., Barth, A. J. \& Matheson, T. Evidence for asphericity in the type IIn supernova SN 1998S. Astrophys. J. 536, 239-254 (2000)

17. Hillier, D. J. An empirical model for the Wolf-Rayet star HD 50896. Astrophys. J. 63 (suppl.), 965-981 (1987).
18. Ofek, E. O. et al. SN 2009ip: constraints on the progenitor mass-loss rate. Astrophys. J. 768, 47 (2013).

19. Chugai, N. N. Circumstellar interaction in type Ibn supernovae and SN 2006jc. Mon. Not. R. Astron. Soc. 400, 866-874 (2009).

20. Hachinger, S. et al. How much $\mathrm{H}$ and $\mathrm{He}$ is 'hidden' in $\mathrm{SNe} \mathrm{lb} / \mathrm{c}$ ? - I. Low-mass objects. Mon. Not. R. Astron. Soc. 422, 70-88 (2012).

21. Vink, J. S. et al. Wind modelling of very massive stars up to 300 solar masses. Astron. Astrophys. 531, A132 (2011)

22. Gräfener, G., Owocki, S. P. \& Vink, J. S. Stellar envelope inflation near the Eddington limit. Implications for the radii of Wolf-Rayet stars and luminous blue variables. Astron. Astrophys. 538, A40 (2012).

23. Crockett, R. M. et al. The type Ilb SN 2008ax: the nature of the progenitor. Mon. Not. R. Astron. Soc. 391, L5-L9 (2008).

24. Arcavi, I. et al. SN $2011 \mathrm{dh}$ : discovery of a type llb supernova from a compact progenitor in the nearby galaxy M51. Astrophys. J. 742, L18 (2011)

25. Bersten, M. C. et al. The type llb supernova $2011 \mathrm{dh}$ from a supergiant progenitor. Astrophys. J. 757, 31 (2012).

26. Van Dyk, S. D. et al. The progenitor of supernova $2011 \mathrm{dh}$ has vanished. Astrophys.J. 772, L32 (2013).

27. Ergon, M. et al. Optical and near-infrared observations of SN 2011dh - the first 100 days. Astron. Astrophys. 562, A17 (2014).

28. Arcavi, I. et al. Core-collapse supernovae from the Palomar Transient Factory: indications for a different population in dwarf galaxies. Astrophys. J. 721, 777-784 (2010).

29. Yaron, O. \& Gal-Yam, A. WISeREP_an interactive supernova data repository. Proc. Astron. Soc. Pacif. 124, 668-681 (2012).

Acknowledgements This research was supported by the I-CORE programme 'The Quantum Universe' of the Planning and Budgeting Committee and The Israel Science Foundation. A.G.-Y. acknowledges support by grants from the ISF, BSF, GIF, Minerva and FP7/ERC, and a Kimmel Investigator award. M.M.K. acknowledges support from Hubble and Carnegie-Princeton fellowships. E.0.O. acknowledges the Arye Dissentshik Career Development Chair and a grant from the Israeli MOST. J.C.W. is supported in part by the NSF. J.M.S. is supported by an NSF Postdoctoral Fellowship. A.V.F. acknowledges financial support from the TABASGO Foundation, the Richard and Rhoda Goldman Fund, the Christopher R. Redlich Fund and the NSF. The National Energy Research Scientific Computing Center, supported by the Office of Science of the US Department of Energy, provided staff, computational resources and data storage for this project. The Oskar Klein Centre is funded by the Swedish Research Council. We thank K. Clubb, O. Fox, P. Kelly, S. Tang and B. Sesar for their help with observations, and J. Groh, P. Crowther, M. Bersten, C. Fransson and E. Nakar for advice. Some of the data presented here were obtained at the W. M. Keck Observatory, which is operated as a scientific partnership among the California Institute of Technology, the University of California and NASA. The observatory was made possible by the generous financial support of the W. M. Keck Foundation.

Author Contributions A.G.-Y. initiated the study, conducted analysis and wrote the manuscript. I.A. found the supernova, triggered rapid follow-up spectroscopy and contributed to light-curve analysis, observations, data reduction and manuscript preparation. E.O.O. contributed to analysis of early-time data, mass loss estimates, temperature evolution and manuscript preparation. S.B.-A. contributed to data reduction and to early light-curve and spectroscopic analysis. S.B.C. reduced Swift and Palomar 60-inch data, and contributed to spectroscopic reduction and analysis. M.M.K. provided APO data and contributed to manuscript preparation. Y.C. contributed to APO data reduction, early light-curve analysis and manuscript preparation. O.Y. contributed to observations and manuscript preparation. D.T. provided unpublished supernova light-curve templates and contributed to photometric analysis. J.M.S. provided spectroscopic reduction and advice, and contributed to HET spectroscopy. A.H. provided early Keck spectroscopy. A.D.C. contributed to observations and data reduction. F.T. reduced NOT data. J.S. provided NOT spectroscopy. D.P. provided Keck spectroscopy and conducted analysis. P.M.V. assisted with observations, spectroscopic analysis, and figure and manuscript preparation. P.E.N. is a PTF builder and contributed to the manuscript. S.R.K. is a PTF builder. A.V.F. provided Keck data and edited the manuscript. J.C.W. provided HET data.

Author Information Reprints and permissions information is available at www.nature.com/reprints. The authors declare no competing financial interests. Readers are welcome to comment on the online version of the paper. Correspondence and requests for materials should be addressed to A.G.-Y. (avishay.gal-yam@weizmann.ac.il). 


\section{METHODS}

Photometry. $r$-band images were obtained by the iPTF survey camera mounted on the Palomar 48-inch Schmidt telescope $\mathrm{e}^{11,30}$. Photometry was measured using our custom pipeline performing PSF photometry on iPTF images after removing a reference image constructed from pre-explosion data using image subtraction Swift ultraviolet absolute AB magnitudes (Extended Data Fig. 1) were measured using standard pipeline reduction ${ }^{31}$ and were corrected for host-galaxy contamination using late-time Swift images.

Spectroscopy. Our earliest (15.5-h) and latest (69-d) spectra were obtained using the Deep Imaging Multi-Object Spectrograph $\left(\right.$ DEIMOS $\left.^{32}\right)$ spectrograph mounted on the Keck II 10-m telescope using the 600 line $\mathrm{mm}^{-1}$ grating and an exposure time of $600 \mathrm{~s}$. Additional spectra were obtained using ALFOSC mounted on the 2.56-m NOT telescope, LRS mounted on the 10.4-m HET telescope (day 3) and the Low Resolution Imaging Spectrometer ${ }^{33}$ (LRIS) mounted on the Keck I 10-m telescope (day 6). All spectra were reduced using standard pipelines and are digitally available on WISeREP ${ }^{29}$.

Spectroscopic observations are presented in Fig. 1. In Fig. 1a, the early spectrum of SN 2013cu reveals Wolf-Rayet wind features. The spectrum (black) is compared with WNL models ${ }^{7}$ (red and grey curves, offset vertically for clarity) showing remarkable similarity, both in line features (major species marked; strong He and $\mathrm{N}$ lines accompanied by Balmer lines indicate a WN6(h) classification) and in the continuum shape (demonstrated by overplotting the $56-\mathrm{kK}$ model on the spectrum). The similarity in continuum shape to hot model spectra constrains any dust reddening to be minimal, indicating that any pre-existing circumstellar dust must have been destroyed; compare with the observed spectrum of the WN6(h) star HD 192163 (blue). Consistent with this conclusion, we detect no trace of $\mathrm{Na}$ D absorption lines. Figure $1 \mathrm{~b}$ shows the emission line evolution during the first week. We replot the first spectrum (red), compared with spectra obtained $\sim 3 \mathrm{~d}$ after explosion (using ALFOSC (blue) and LRS (cyan)) and $\sim 6 \mathrm{~d}$ after explosion (magenta additional details in A.G.-Y. et al., manuscript in preparation). Spectra were scaled and offset for clarity with respect to the high-quality Keck/LRIS spectrum. Continuum shapes are identical (within calibration uncertainties) and consistent with the Rayleigh-Jeans tail of a hot Planck curve, indicating that the black-body peak remains in the ultraviolet at $6 \mathrm{~d}$ past explosion. By day 3 , the initially strong WolfRayet features disappear, with the exception of weaker He II lines and the C III/N III complex around $4,640 \AA$ (marked), whereas the $\mathrm{H} \alpha$ line remains constant at $3.4 \times$ $10^{-15} \mathrm{erg} \mathrm{s}^{-1} \mathrm{~cm}^{-2}$ (with decreased equivalent width due to higher continuum level). The spectrum with a high signal-to-noise ratio on day 6 is almost featureless, except for weak $\mathrm{H} \alpha$ emission (inset) with an intensity less than a tenth that of day 3 , probably because the line-forming region has been cleared by the expanding ejecta. Figure 1c shows evidence that SN 2013cu is of type IIb. A spectrum of SN 2013cu $69 \mathrm{~d}$ after explosion (black) is compared with the prototypical type IIb supernovae SN 1993J at age $60 \mathrm{~d}$ (red) and SN 2011dh at age $43 \mathrm{~d}$ (green), and with the typical non-stripped type II-P supernova SN 2004et at age $45 \mathrm{~d}$ (blue). To allow for slight age differences and expansion-velocity variations, we align all spectra in wavelength using the weak blue-shifted Fe II lines at 4,924, 5,018 and 5,169 (marked with black vertical lines), because they are good tracers of the photosphere. Supernovae SN 2013cu, SN 2011dh and SN 1993J exhibit strong He I absorption at 5,876, 7,065 and $7,281 \AA$ (marked with black vertical lines), whereas the weaker 6,678 $\AA$ absorption is similar in SN 2013cu and SN 2011dh. These He I lines are not detected in the type IIP supernova spectrum at all. However, SN 2013cu shows weaker Balmer absorption (marked with magenta vertical lines), and the $\mathrm{H} \alpha$ absorption is not clearly defined. Both the spectral similarity to supernovae SN 1993J and SN 2011dh and the strong $\mathrm{He}$ lines relative to $\mathrm{H}$ indicate that $\mathrm{SN} 2013 \mathrm{cu}$ is spectroscopically a type IIb supernova. Flash spectroscopy. We define as 'flash spectroscopy' the technique of obtaining a set of spectroscopic data shortly enough after a supernova explosion that the observed spectrum is dominated by features related directly to the effects of the shock breakout flash. In particular, flash-ionized CSM recombines and forms strong emission lines, revealing, for example, the elemental abundance and, thus, the Wolf-Rayet class of a supernova progenitor. In addition, emission line spectra provide a handle on the early temperature evolution, which is difficult to measure even using Swift ultraviolet photometry because the black-body peak is initially too far into the ultraviolet. This study provides strong motivation for future investigations using dedicated rapid-response spectrographs, such as FLOYDS ${ }^{3,34}$ and $\mathrm{SEDM}^{35}$, responding to real-time triggers from high-cadence wide-field surveys ${ }^{12}$.

Although Wolf-Rayet supernova progenitor stars are difficult to study using pre-explosion imaging (owing both to intrinsic low luminosity in the optical and infrared bands $\mathrm{s}^{14,36}$ and to the possible confusing effect of a bright $\mathrm{O} / \mathrm{B}$ companion $^{3}$ ), we demonstrate that they are amenable to study using the flash spectroscopy method. WRSs belonging to the $\mathrm{WN}(\mathrm{h})$ class may have the most extensive winds ${ }^{1,14}$. Application of this method to WC and WO stars may require flash spectroscopy at even earlier epochs ( $\sim 1 \mathrm{~h}$ after explosion), before the supernova ejecta sweep up the high-density wind. Reducing the latency between the explosion and the spectroscopy by an order of magnitude relative to our observations of SN 2013cu is possible using recently commissioned instrumentation (Extended Data Fig. 2).

Unlike studies of supernova progenitors through pre-explosion imaging, the flash spectroscopy method can be applied to relatively distant objects (SN 2013cu is located $108 \mathrm{Mpc}$ away, well beyond the 20-Mpc distance typical for pre-explosion studies) and to events in galaxies having no high-quality pre-explosion imaging, such as a large population of little-studied dwarf galaxies. Judging from local supernova rate measurements ${ }^{37}, \sim 300$ explosions occur within $100 \mathrm{Mpc}$ of Earth every year and can be potentially studied in this manner. The method thus allows routine spectroscopic studies of supernova progenitors that were previously only possible by extreme serendipity (for example for the progenitor of SN 1987A; ref. 38). Within a few years, there will be enough data for the flash spectroscopy method to be used to chart wind signatures from numerous supernova progenitors; in particular, it will be possible to study systematically the Wolf-Rayet progenitor population of stripped supernovae. We thus expect that this method will be broadly applied in the coming years.

30. Rau, A. et al. Exploring the optical transient sky with the Palomar Transient Factory. Proc. Astron. Soc. Pacif. 121, 1334-1351 (2009).

31. Poole, T. S. et al. Photometric calibration of the Swift ultraviolet/optical telescope. Mon. Not. R. Astron. Soc. 383, 627-645 (2008).

32. Faber, S. M. et al. The DEIMOS spectrograph for the Keck II Telescope: integration and testing. Proc. SPIE 4841, 1657-1669 (2003).

33. Oke, J. B. et al. The Keck Low-Resolution Imaging Spectrometer. Proc. Astron. Soc. Pacif. 107, 375-385 (1995).

34. Ben-Ami, S. et al. Discovery and early multi-wavelength measurements of the energetic type Ic supernova PTF12gzk: a massive-star explosion in a dwarf host galaxy. Astrophys. J. 760, L33 (2012).

35. Ben-Ami, S. et al. The SED machine: a dedicated transient IFU spectrograph. Proc. SPIE 8446, 844686 (2012).

36. Yoon, S.-C., Gräfener, G., Vink, J. S., Kozyreva, A. \& Izzard, R. G. On the nature and detectability of type lb/c supernova progenitors. Astron. Astrophys. 544, L11 (2012).

37. Li, W. et al. Nearby supernova rates from the Lick Observatory Supernova Search II. The observed luminosity functions and fractions of supernovae in a complete sample. Mon. Not. R. Astron. Soc. 412, 1441-1472 (2011).

38. Walborn, N. R. et al. The spectrograms of Sanduleak -690202 , precursor to supernova 1987A in the Large Magellanic Cloud. Astron. Astrophys. 219, 229-236 (1989).

39. Niemela, V. S., Ruiz, M. T. \& Phillips, M. M. The supernova 1983k in NGC 4699 - clues to the nature of type II progenitors. Astrophys. J. 289, 52-57 (1985).

40. Phillips, M. M. et al. The light curve of the plateau type II SN 1983K. Proc. Astron. Soc. Pacif. 102, 299-305 (1990) 




Extended Data Figure $1 \mid$ The $r$-band light curve of SN 2013cu. A parabolic model of the flux-time (red solid curve) describes the pre-peak data ( $1 \sigma$ error bars) very well. Backward extrapolation indicates an explosion date of UTC 2013 May 2.93 \pm 0.11 (MJD $=56414.93 ; 5.7 \mathrm{~h}$ before the first iPTF detection; see inset); we estimate the uncertainty from the scatter generated by modifying the subset of points used in the fit. Our first Keck spectrum was obtained about $15.5 \mathrm{~h}$ after explosion (vertical dotted line). Early Swift ultraviolet photometry (diamonds) places a lower limit of $T=25,000 \mathrm{~K}$ on the black-body temperature measured $40 \mathrm{~h}$ after explosion. 


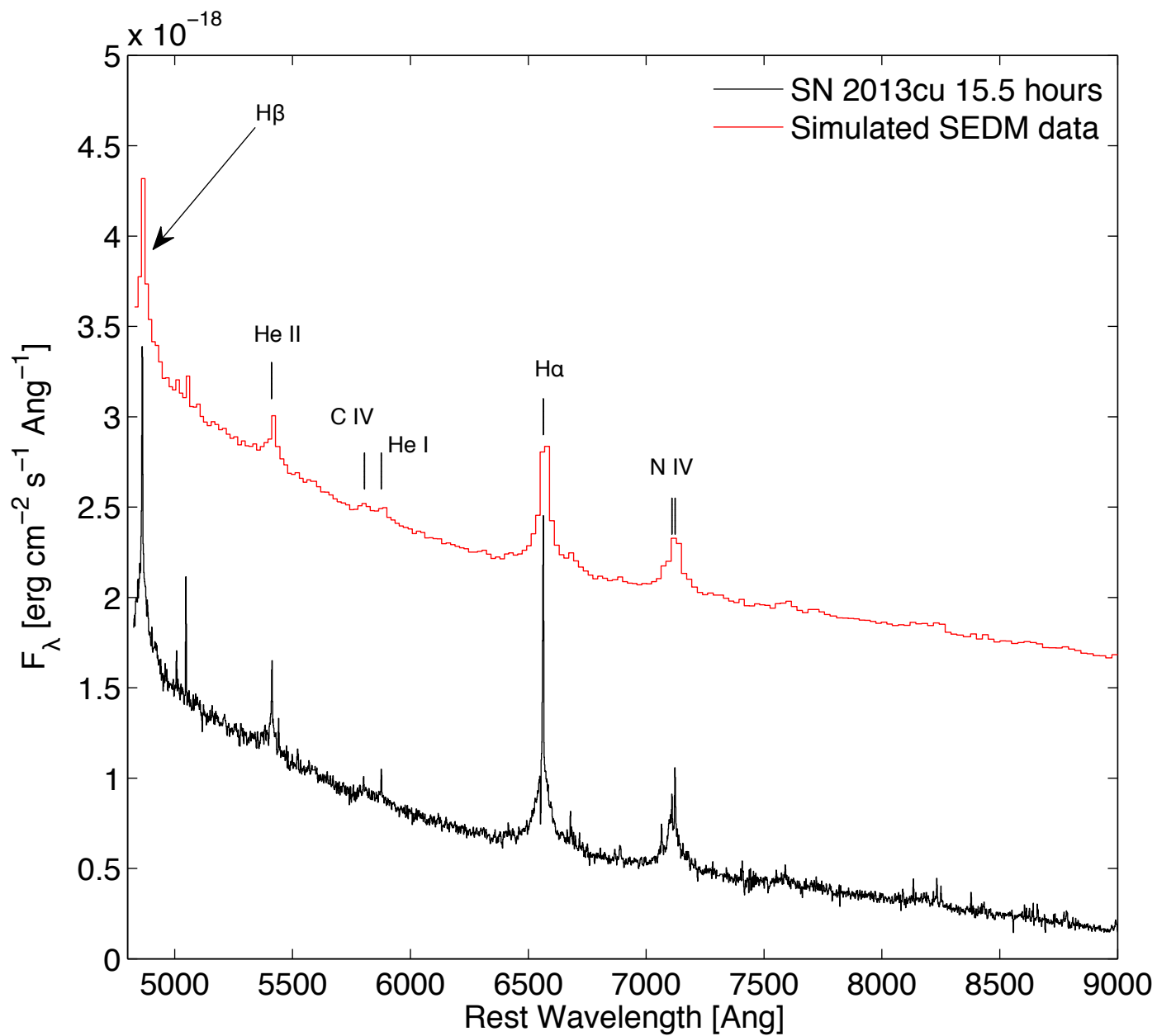

Extended Data Figure $2 \mid$ Flash spectroscopy: rapid spectroscopic observations of supernovae during or shortly after shock breakout. This simulated SEDM spectrum (red) created by downgrading the observed Keck spectrum (black; resolution, $R=2,000$ ) to the coarse SEDM resolution $(R=100)$ shows that the strong Wolf-Rayet lines (in this case the marked $\mathrm{H}$, $\mathrm{He}$ and $\mathrm{N}$ lines) are still easily detectable and allow us to determine the WolfRayet spectroscopic class. The SEDM is an IFU low-resolution spectrograph designed for robotic response to transient events, to be mounted on the Palomar 60-inch telescope almost continually. Responding to real-time triggers from the iPTF survey operating on the same mountain, this instrument should be able to obtain low-resolution spectra within $\sim 1 \mathrm{~h}$ of object detection. Because SEDM operates on a smaller telescope than Keck, SEDM data of similar quality to the simulated spectrum will require a relatively long integration. However, SEDM will be able to observe objects with much reduced latency, thus benefiting from stronger line intensities expected from the stronger shock breakout flash luminosity processed by a denser wind close to the progenitor star, potentially compensating for its reduced absolute sensitivity. 


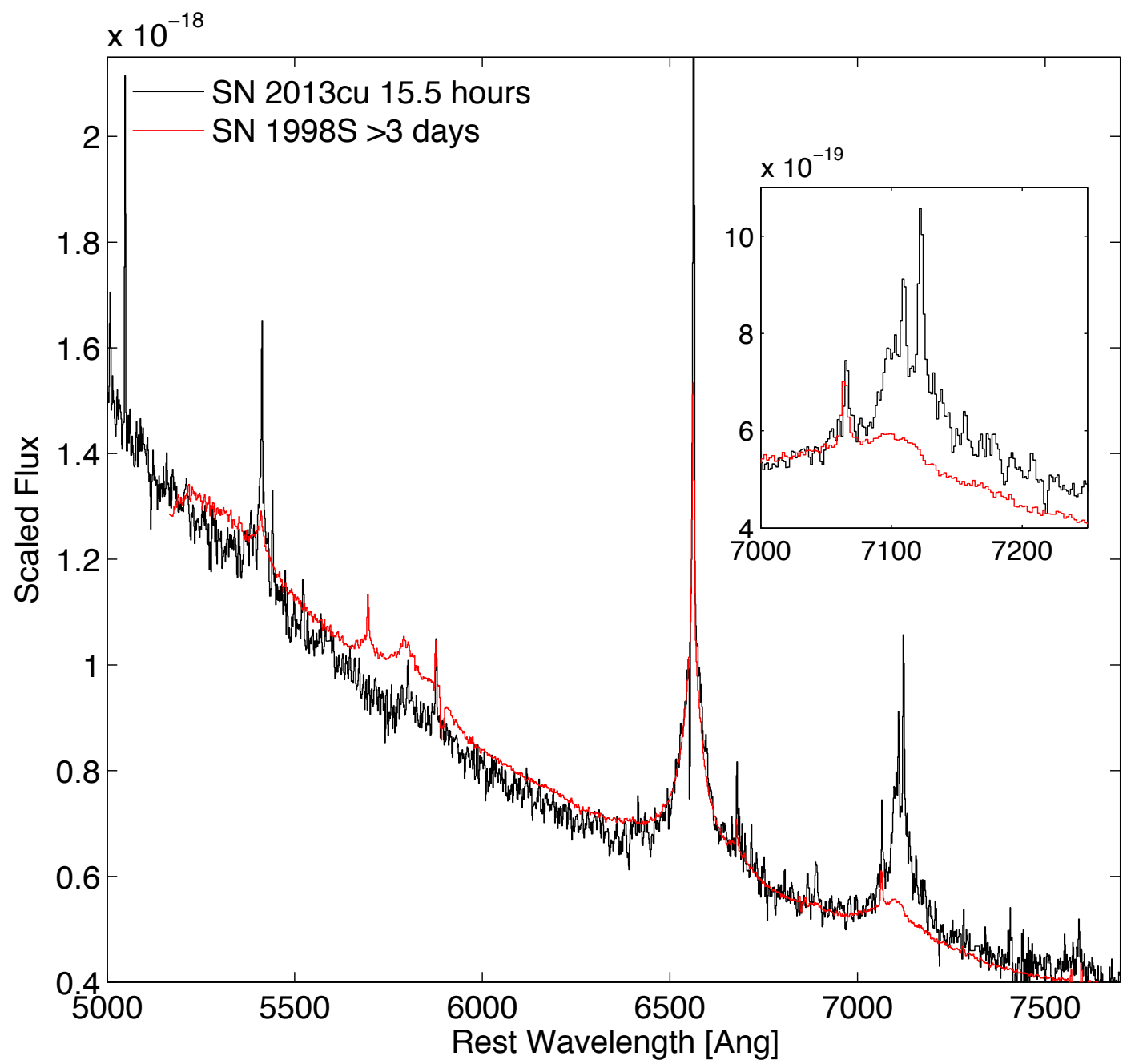

Extended Data Figure 3 Comparison with early 'Wolf-Rayet' spectra of SN 1998S. Wolf-Rayet-like features similar to those we observed were previously noted in two cases, SN 1983K (refs 39,40) and SN 1998S (ref. 16), and persisted for many days after explosion. The spectra of SN 1983K, classified as a type II-P supernova, are unfortunately not available for comparison. Spectra of SN 1998S (type IIn) are shown here. The spectra have a similar blue continuum slope and a similar $\mathrm{H} \alpha$ profile. The He II $\lambda 5,411$ and the $\mathrm{N}$ IV $\lambda 7,115$ complex are weaker in SN 1998S, and the strong lines of N and C $(5,806 \AA)$ are broad, consistent with an origin in shocked $\mathrm{CSM}^{16}$ rather than in an undisturbed Wolf-Rayet wind. The inset shows a close-up view of the strong $\mathrm{N}$ IV 27,115 complex. N emission from SN $1998 \mathrm{~S}$ is weak compared with He I $\lambda 7,065$ and shows a smooth, broad profile, whereas SN 2013cu exhibits a broad base (full-width at half-maximum, $2,500 \mathrm{~km} \mathrm{~s}^{-1}$ ) as well as strong and narrow (unresolved) N IV lines. These observations are consistent with an origin for the $\mathrm{N}$ emission of SN 1998 S in shocked (and perhaps N-rich) CSM ${ }^{16}$, whereas the narrow He I lines may come from a more distant, photo-ionized wind. In SN 2013cu even the narrow $\mathrm{N}$ lines are much stronger than $\mathrm{He}$, indicating a wind with a Wolf-Rayet-like composition at all radii. 